\title{
Instrumental Quality Control of Therapeutic Linear Accelerator Performance
}

\author{
Mohammed Ahmed Ali Omer ${ }^{1,2}$ \\ ${ }^{1}$ Department of Radiologic Technology, College of Applied Medical Science, Qassim University, Buraidah, KSA \\ ${ }^{2}$ Department of Radiotherapy \& Nuclear Medicine, College of Medical Radiologic Science, Sudan University of Science and Technology, \\ Khartoum, Sudan
}

Email address:

alkajam@gmail.com

\section{To cite this article:}

Mohammed Ahmed Ali Omer. Instrumental Quality Control of Therapeutic Linear Accelerator Performance. American Journal of Physics and Applications. Vol. 5, No. 5, 2017, pp. 66-72. doi: 10.11648/j.ajpa.20170505.12

Received: July 19, 2017; Accepted: July 28, 2017; Published: August 16, 2017

\begin{abstract}
The objective of the article was to assess therapeutic linear accelerator performance. Material \& method used were quality control tools, direct measurement \& theoretical calculation methods. The analysis of results showed that: shift of machine isocenter was $1 \mathrm{~mm}$ then increases up to $2 \mathrm{~mm}$ through the gantry angles 0 to $300^{\circ}$ and 300 to 360 respectively. The diaphragm rotation isocenter clock \& anti-clock wise was $1 \mathrm{~mm}$. the light and radiation fields showed concise matching up to $9 \times 9 \mathrm{~cm}$, then for $10 \times 10,14 \times 14$ and $16 \times 16 \mathrm{~cm}$ there were incongruence by $0.25,0.3$ and $0.41 \mathrm{~cm}$ respectively. The increment of the field sizes $(2 \times 2,4 \times 4-20 \times 20) \mathrm{cm}$ following SSD increment fitted with the inverse square law significantly $\left(\mathrm{R}^{2}=1\right)$. The theoretical (calculation method) field size was greater than the measured (practical) field size relative to SSD by $0.2 \mathrm{~cm}$. The system output in $\mathrm{Gy} / \mathrm{Mu}$ increases significantly $\left(\mathrm{R}^{2}=0.9\right)$ as the field size increases in logarithmic equation; while it decreases as SSD increases. The measured output on phantom surface was greater $(0.8 \mathrm{~Gy} / \mathrm{MU})$ than that calculated theoretically which was $(0.5 \mathrm{~Gy} / \mathrm{MU})$. A significant $\left(\mathrm{R}^{2}=0.8\right)$ reduction in output reading following the increment of temperature for Linac 10 MV and $6 \mathrm{MV}$ respectively, while the pressure lead to significant (0.6) increment of system output reading. TLD showed narrow penumbra extension as 0.32 and $0.2 \mathrm{~cm}$ for lianc $6 \mathrm{MV}$ and $10 \mathrm{MV}$ respectively compared with 0.5 and $0.3 \mathrm{~cm}$ at maximum depth dose when obtained from dose histogram.
\end{abstract}

Keywords: Linear Accelerator, Accuracy, Quality Control, Radiotherapy, Instrumental

\section{Introduction}

The linear accelerator (Linac) has been one of the most favorable and commonly used in developed countries as radiation teletherapy machine offering dual function as electron and x-ray photons, hence leading for eclipsing of ${ }^{60}$ Co-teletherapy machine among these countries, while ${ }^{60} \mathrm{Co}$-teletherapy machine commonly utilized in developing countries. Linacs have been used intensively in irradiation of tumors (benign and malignant), however although it is high accuracy in mounting and installation; it seeks for quality control (QC) for the purposes of constancy and consistency related to delivered tumor dose that should not exceeds $\pm 5 \%$ of the prescribe dose $[1,2]$ or the target dose should be controlled within $5-7 \%$ of the prescribed dose as reported by ICRU report 50 [3]. Out of practical experience,
Linacs have minimum faults or in-accuracy in its components although it is so sensitive to variability of temperature, pressure and any mishandling or dust; causing common errors which in turn interrupting the treatment sessions specially in developing countries. The source of errors have been increases as the high technology and complexity of the system being adapted, hence the need for QC and routine inspection are inevitable to trigger and classify the tolerance and action levels before errors have an impact on patient care [4], as it has been noted that implementing of QA program in radiotherapy will contribute in prevention of systematic errors as well as reducing the random errors [5-7]. In relation to such trend; many parameters could influence the accuracy of radiotherapy plan, for instance: variation in radiation field size (FS), source to surface distance SSD, system output, environmental factors affecting radiation dosimetry 
(temperature and pressure), laser beams of patient setup, machine isocenter and the beam distribution with relative penumbra profile which are all in turn will be under focus and as a trend of this study. The density of atmospheric air depends upon the following factors: Composition, Moisture content, Temperature, and Pressure; and with considering these factors; air composition is extremely uniform for altitudes up to $15 \mathrm{~km}$. and over the earth's surface. While the ordinary variations in density resulting from change in composition do not affect the ionization produced in air. A change in temperature of $10^{\circ} \mathrm{C}$ causes a change in air density due to its moisture content of only about one-half per cent, therefore the air density changes are due to variations in temperature and pressure and in turns it is density (unconfined air) varies inversely as its absolute temperature and directly as its pressure based in equation (1) [8], therefore the Rontgen is uniquely defined when these conditions are given and to fulfill this requirement the definition states that the ionization shall be measured in air at a temperature of $0^{\circ} \mathrm{C}$ and a pressure of $760 \mathrm{~mm}$ mercury and the correction of density $d$ at $0^{\circ} \mathrm{C}\left(273^{\circ}\right.$, absolute $)$ and
$760 \mathrm{~mm}$ mercury may be calculated from the density $d_{\mathrm{t}}$ at temperature $t^{\circ} \mathrm{C}(273+\mathrm{t}$, absolute $)$ and pressure $p$ by the equation (2):

$$
\text { Density } \alpha \frac{p}{t}
$$

Where $p$ is the pressure and $t$ refers to temperature.

$$
d_{0}=d_{t}\left(\frac{273+t}{273} \times \frac{760}{p}\right)
$$

The correction of temperature and pressure in radiation dosimetry and radiotherapy should be serious; although the studies showed that: a variation in temperature by $\left( \pm 3^{\circ} \mathrm{C}\right)$ that corresponds to air density change would result in an error of \pm 1 per cent and as well the variation of atmospheric pressure of $\pm 10 \mathrm{~mm} \mathrm{Hg}$ will introduce an error of about \pm 1 per cent [9]. However this error could be maximized due to other errors percent occur during the stages of radiotherapy plan. Hence the trend of this study will focus on the mechanical and dosimetric quality control tests of linear accelerator:

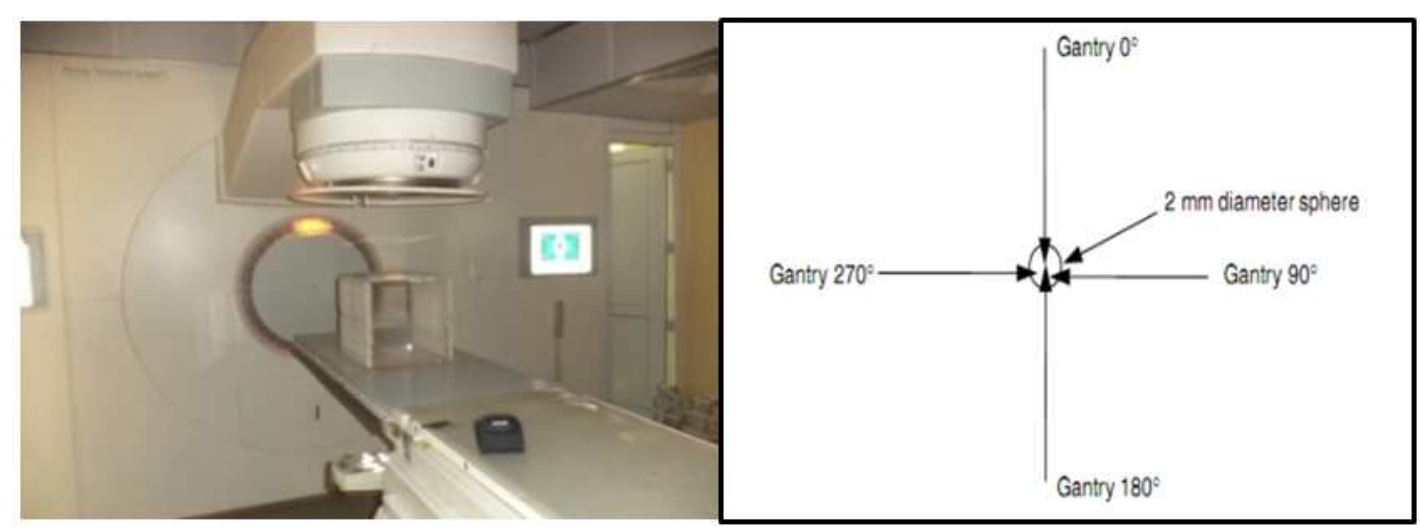

Figure 1. Shows the set up for assessing diaphragm rotation accuracy, and gantry rotation isocenter.

\section{Methodology}

All mechanical tests done above according to AAPM and Swiss Society of Radiobiology and Medical Physics Member of the European Federation of Organizations for Medical Physics (EFOMP) and the International Organization for Medical Physics (IOMP) Recommendations No. 11 November 2003.

\subsection{Accuracy of Gantry Isocenter}

The gantry isocenter is the smallest sphere through which the axis of the radiation beam pass at all gantry angles and it should be consider from the first point of system installation by the company vendor as quality assurance step. It has been done by setting the gantry at $0^{\circ}$, and driving the treatment couch to the maximum outwards, then fixing a pin at it is edge; so that the projected cross-hair shadow is shining in concise with the pin; then both shadows of cross-hair and the pin were received on a graphic paper which assuming that: the shadows are superimposed at different gantry angles; and if any deviation appeared, should not exceeds $2 \mathrm{~mm}$.

\subsection{Diaphragm Rotation}

This test was performed prior to other tests of the mechanical isocenter which are based on the assumption of correctly aligned crosshairs. To assess diaphragm rotation isocenter (Rotation axis of the Collimator), the field size of $10 \times 10 \mathrm{~cm}^{2}$ has been projected on graphic paper using source skin distance SSD $=100 \mathrm{~cm}$, and the field center has been defined by the shadow of transistor shape over the graphic paper Figure 1, with the gantry and treatment table angles set to zero then rotate the diaphragm angles clock wise (negative degrees) and anti-clock wise (positive degrees) to the 0,45 , $90,135,180 \ldots 275^{\circ}$ in order to establish the maximum deviation with collimator rotation, the plotting of angles versus shift in mm has been done. The frequency of the test is monthly, and the permissible deviation should be within 2 $\mathrm{mm}$ diameter circle containing the isocenter which was called tolerance.

\subsection{Light and Radiation Field Coincidence}

To test the congruence of the radiation field and light field; 
an enveloped film is placed perpendicular to the beam central axis at the isocenter. The edges of the light field and the crosshair are marked on the top of enveloped film according to the projection of light field and the cross-hair and a pin punctures were done at edges as shown in Figure 2. Then a corresponding buildup embedded on the film, adjusting the $\mathrm{SSD}$ at $100 \mathrm{~cm}$ and exposing the film. The film processed and measurements have been done to compare between the extension of light field based on the puncture sides and the edge profile of the radiation as well as the variation of the field center. The variation or accepted limit should not exceed $2 \mathrm{~mm}$ for field sizes $<20 \times 20 \mathrm{~cm}^{2}$ at standard SSD. Where $\mathrm{W}_{\mathrm{T}}$ refers to weighting factor for organ or tissue and $\mathrm{H}_{\mathrm{T}}$ refers to equivalent dose to organ or tissue.

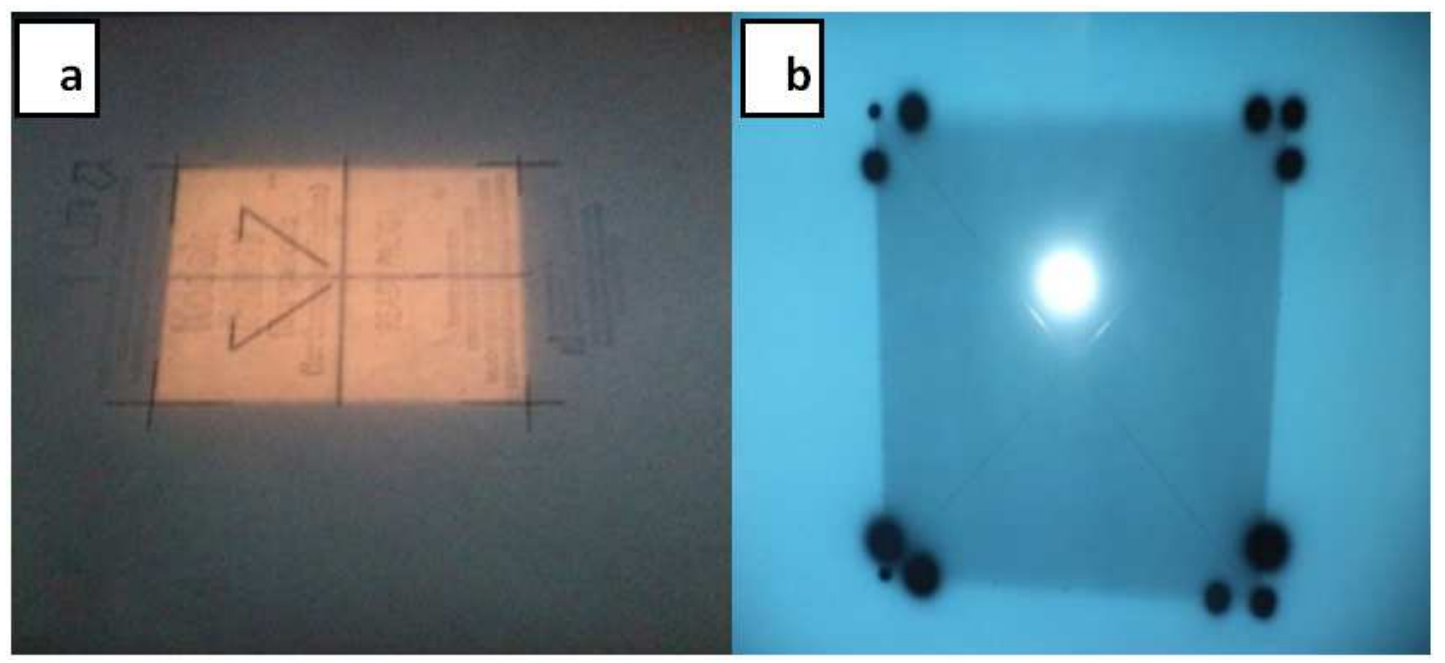

Figure 2. Shows the setup configuration of light and radiation fields' coincidence (a) the enveloped film (none screen film) (b) the processed film with puncture marks for light field.

\subsection{Method of Theoretical and Practical Field Size}

To carry out such correlation, different field sizes of $20 \times 20,14 \times 14,12 \times 12,10 \times 10,8 \times 8,6 \times 6,4 \times 4,2 \times 2 \mathrm{~cm}^{2}$ with different SSD $=90,95,100,105,110,115,120 \mathrm{~cm}$ have been adjusted and the relevant light fields have been calculated based on the inverse square law and from the measurement of light field projected over the graphic paper and consequently the SSD decreased from $100 \mathrm{~cm}$ to 95,90 $\mathrm{cm}$ then from 100 to $105,110,115,120$ and the relevant field sizes have been measured and calculated.

\subsection{Assessment of Penumbra Profile}

The penumbra represents irradiated profiles around the actual radiation field due to large radiation source or extended SSD. It has been assessed from setting a field size of $10 \times 10 \mathrm{~cm}$ at $\mathrm{SSD}=100 \mathrm{~cm}$ and determining the system energy then applying a treatment planning system that using software (ominipro) to obtain dose histogram with relevant isodose curves, then the penumbra profile has been determine and measured from the beam profile between $80 \%$ and $20 \%$ and at full width at half maximum (WHM).

\subsection{Laser Alignment}

The aim of such test is to verify that all laser beams correctly indicate the isocenter and that opposing laser beams are congruent. To so, a rotatable plate is adjusted in such a way that a point marked on its rotation axis is coincident with the light field crosshair at SAD. It is checked that the lateral and longitudinal lasers are coincident with this mark as shown in Figure 3.
For congruence of lasers a piece of paper is put into the lateral laser beam at a distance further than $20 \mathrm{~cm}$ from the isocenter. The maximum deviation between the projections of the right and the left lateral lasers is recorded. The projection of the longitudinal laser is compared to a reference mark, for example on the floor, (that is made at the time of acceptance of the laser system). The accepted limit of deviation should not exceed 1-2 $\mathrm{mm}$.

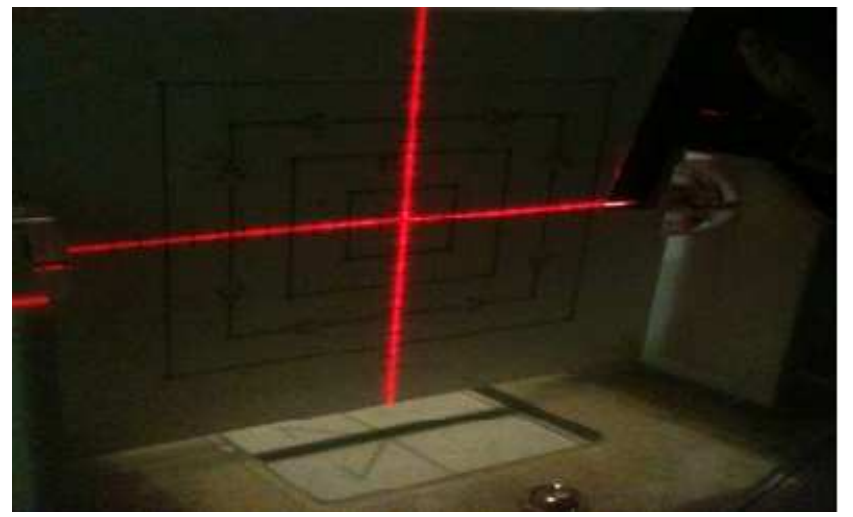

Figure 3. Shows the setup for laser beams coincident quality control in linear accelerator.

\subsection{Dosimetry (Output Measurement)}

According to IAEA TRS 398 protocol for weekly output measurement of high energy photon beam and by setting a field size of $10 \times 10 \mathrm{~cm}^{2}$ at SSD $=95 \mathrm{~cm}$, and at Depth $=5 \mathrm{~cm}$ and by using a FC65-P chamber, water phantom and electrometer to detect the variation of the output beam 
relative to the effect of temperature and pressure as shown in Figure 4.
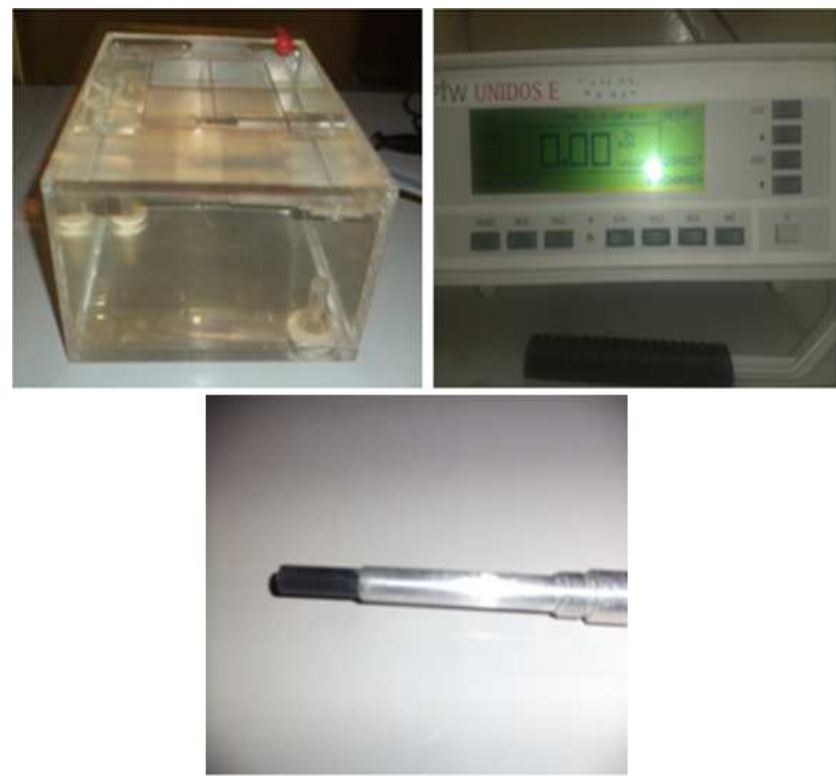

Figure 4. Shows the utilized FC65-P chamber, water phantom and electrometer for linear accelerator output measurement of photon beam.

\section{Results}

The following results show the related findings of quality control tests for linear accelerator performance as mechanical and dosimeric assessments.

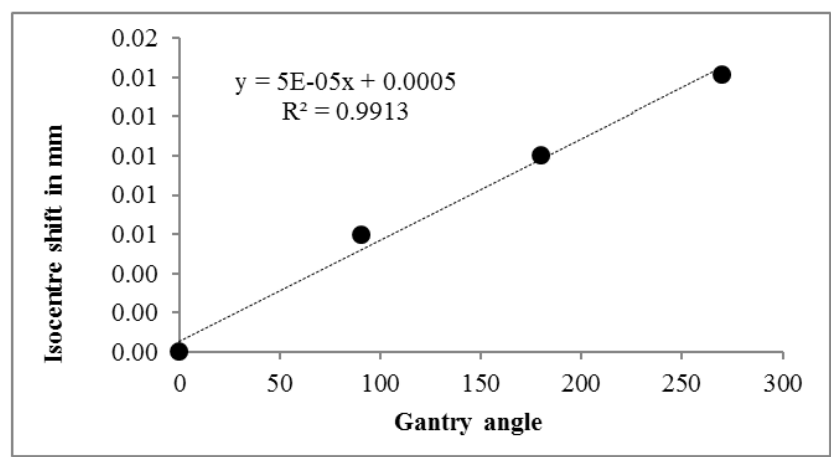

Figure 5. Shows the correlation between the Gantry angle and the machine isocenter shift in $\mathrm{mm}$.

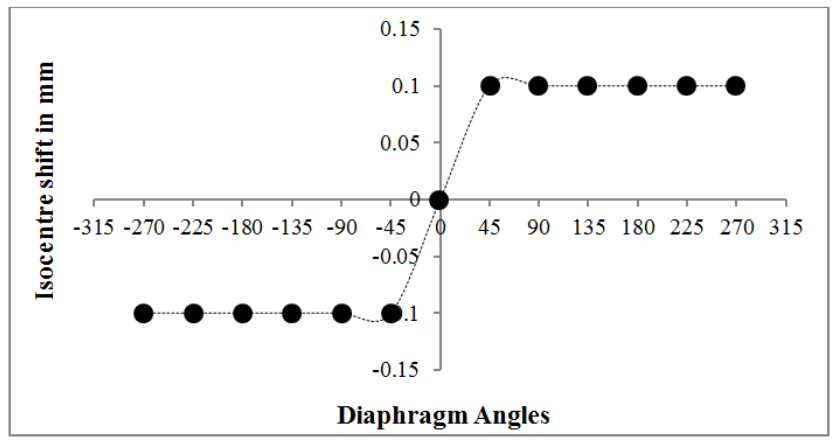

Figure 6. shows the correlation between the diaphragm angles and its isocenter shift in $\mathrm{mm}$.

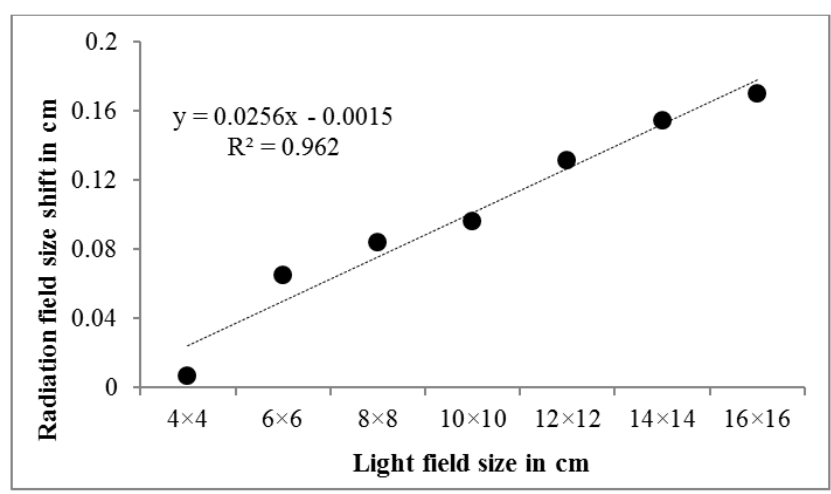

Figure 7. Shows the correlation between the light field and the radiation field shift in $\mathrm{cm}$.

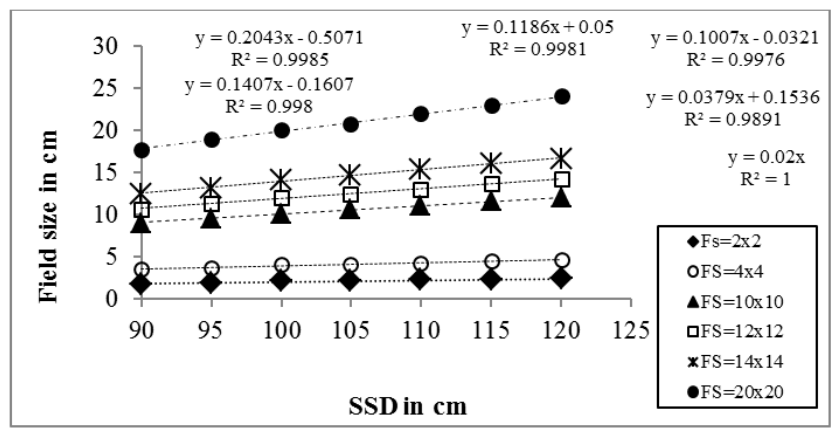

Figure 8. Shows the correlation between the practical field sizes in $\mathrm{cm}^{2}$ and different SSDs in Linear accelerator.

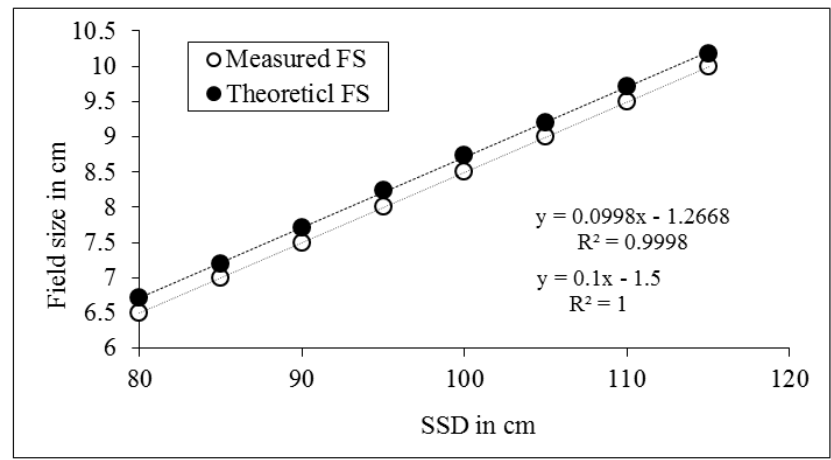

Figure 9. Shows the correlation between the SSD versus the measured (practical) \& theoretical field's size in $\mathrm{cm}$.

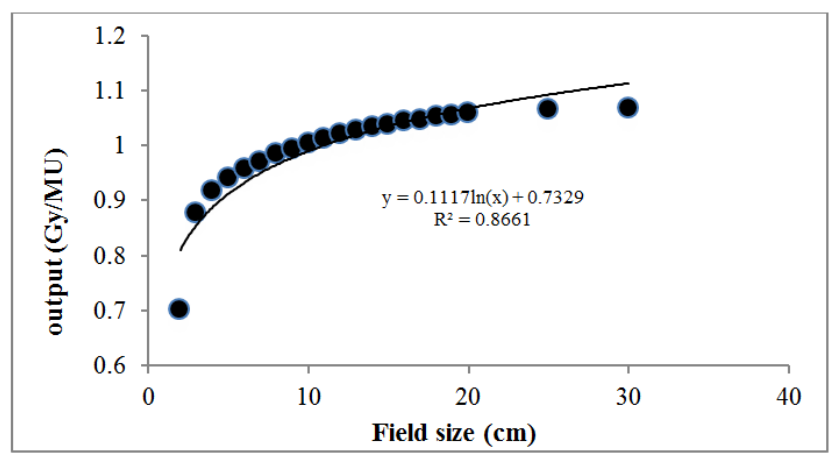

Figure 10. Shows the correlation of output in $\mathrm{Gy} / \mathrm{Mu}$ versus field sizes in $\mathrm{cm}$ at $S S D=100 \mathrm{~cm}$. 


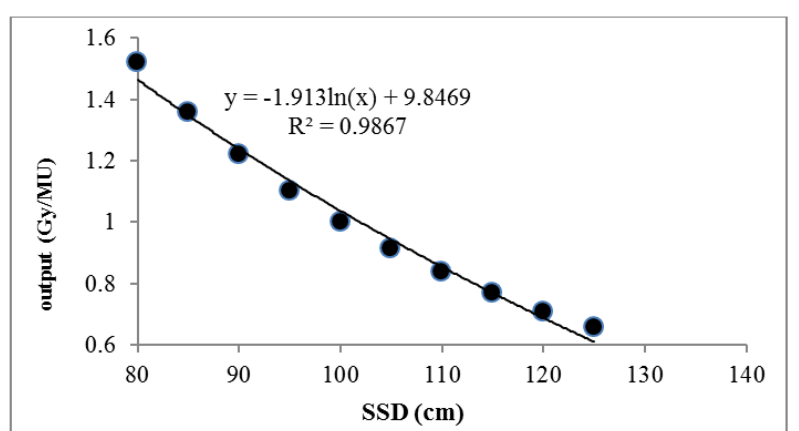

Figure 11. Shows the correlation of output in Gy/Mu versus SSDs at field size of $10 \times 10 \mathrm{~cm}$.

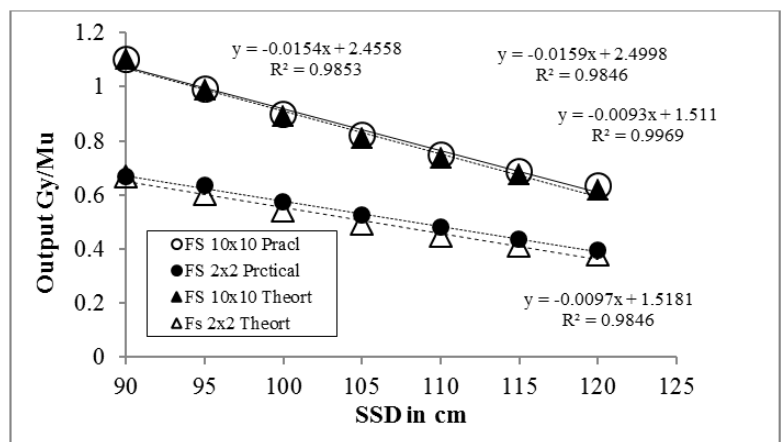

Figure 12. Shows the variation of Linear accelerator output versus SSDs as practical and theoretical measurement.

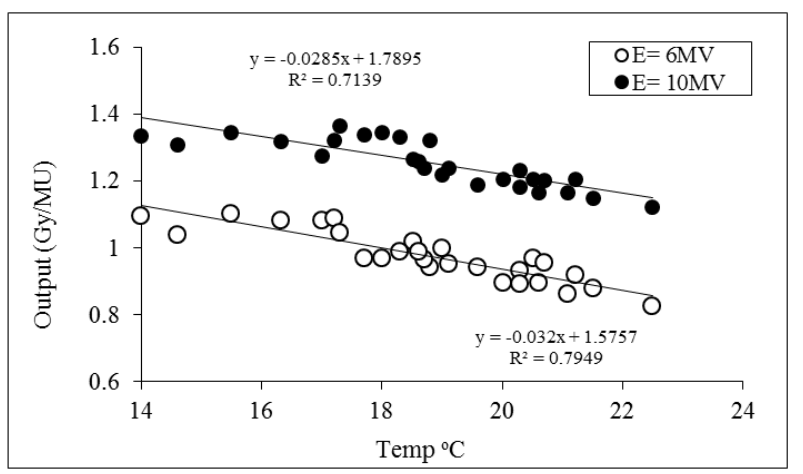

Figure 13. Shows the Linear accelerator 6 and $10 \mathrm{MV}$ output readings versus temperature in ${ }^{\circ} \mathrm{C}$.

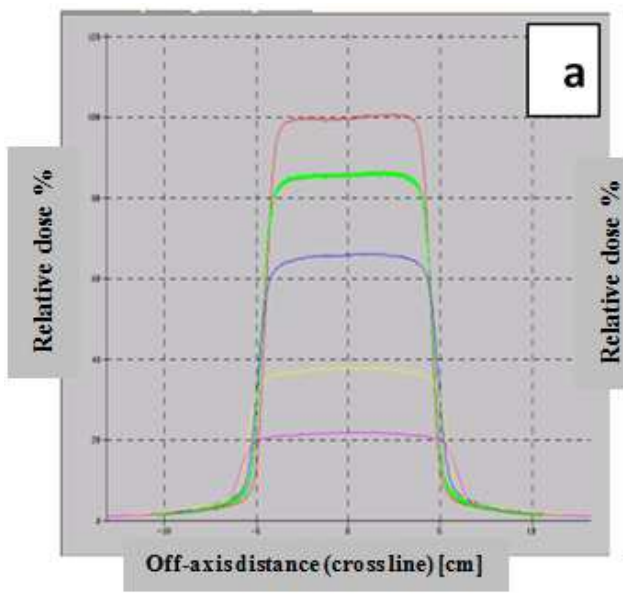

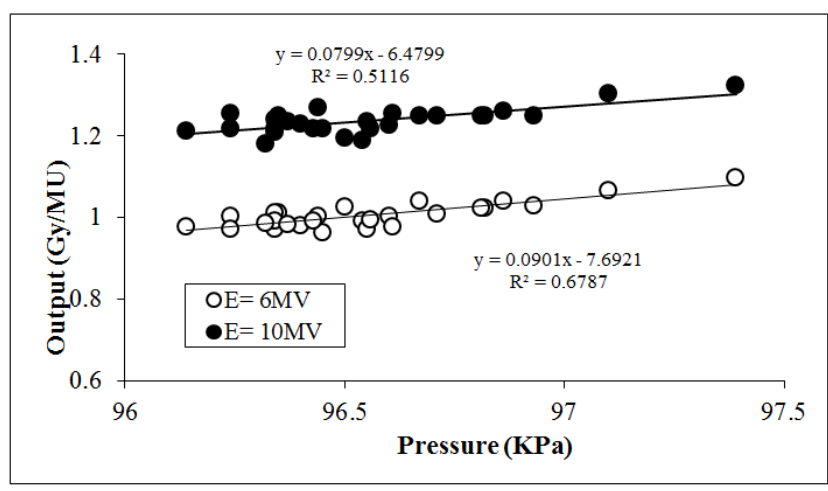

Figure 14. Shows the Linear accelerator 6 and $10 \mathrm{MV}$ output readings versus pressure in Kpa.

\section{Discussion \& Analysis}

Figure 5: shows the significant $\left(\mathrm{R}^{2}=1\right)$ correlation between the Gantry angle and the machine isocenter shift in $\mathrm{mm}$, which revealed that: the angulation of machine gantry accompanied with trifle shift of the isocenter $(0.01)$ from 0 up to $300^{\circ}$ then increases up to $0.02^{\circ}$ between gantry angles 300 to 360 . Such shift has been within the tolerance limit $( \pm 2$ $\mathrm{mm}$ ) as linear accelerator is less coming faults relative to ${ }^{60}$ Co-teletherapy machine.

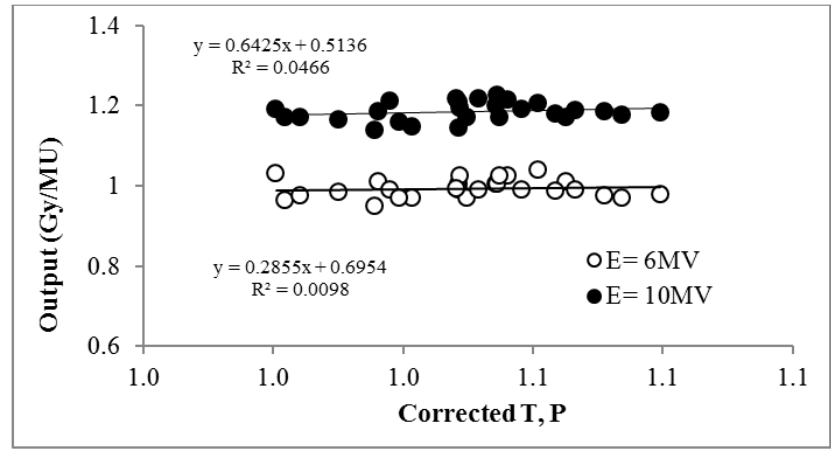

Figure 15. Shows the Linac 6 and 10 MV output readings corrected for temperature and pressure.

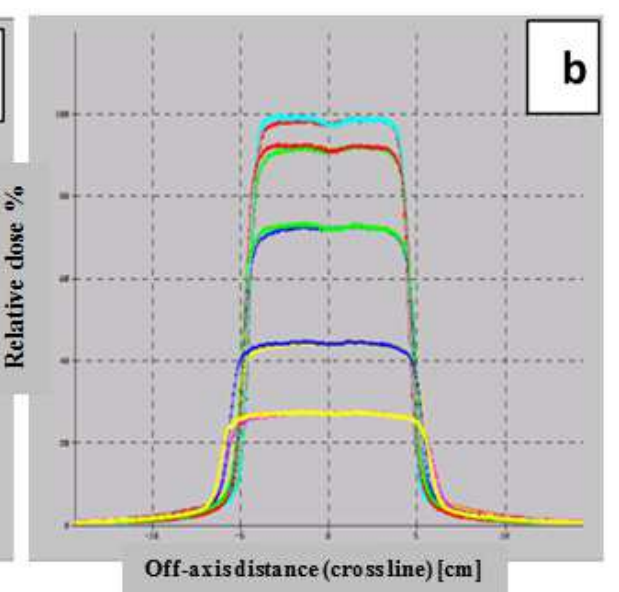

Figure 16. Shows the dose histogram for (a) Linac $6 \mathrm{MV}$ and (b) $10 \mathrm{MV}$ with relative penumbra profiles for a field size $10 \times 10 \mathrm{~cm}$ and Dmax. 
The test of correlation between the diaphragm rotating angles and its isocenter (Figure 6) revealed that: the diaphragm rotation (clock \& anti-clock wise) accompanied with a shift up to $1 \mathrm{~mm}$ and continue constantly which is within the tolerance level $\left( \pm 1^{\circ}\right)$, such defect could be ascribed to either of grove corrosion or ball bearing erosion.

Figure 7: shows the correlation between the light field and the radiation field shift in $\mathrm{cm}$, in which there was an acceptable radiation field shift for the equivalent field sizes up to $9 \times 9 \mathrm{~cm}$, and for the field size $10 \times 10,14 \times 14$ and $16 \times 16$ $\mathrm{cm}$ there was obvious increment of shift $0.25,0.3$ and 0.41 $\mathrm{cm}$ respectively. Same test for assessing the variation of practical (measured) field size versus SSD (Figure 8), showed that: the Field size has been increased linearly with SSD increment in a significant correlation $\left(R^{2}=1\right)$ for the field sizes $(2 \times 2,4 \times 4-20 \times 20) \mathrm{cm}$; however in small field sizes $(2 \times 2)$ and $(4 \times 4) \mathrm{cm}$; the impact of SSD changes was not so obvious relative to the larger field sizes $(10 \times$ $10,12 \times 12,14 \times 14) \mathrm{cm}$ and greater. Such effect could be ascribed to obvious field divergence relative to extended SSD; and of course such effect would be reflected on the delivered radiation dose in case of cancer patient irradiation [10]. Figure 9: shows the correlation between the SSD versus the measured (practical) \& theoretical field's size in $\mathrm{cm}$, which showed that: there were linear proportional significant $\left(\mathrm{R}^{2}=1\right)$ correlations between SSD and both the theoretical and measured field sizes based on equation $y=0.0998 x$ 1.2668 and $y=0.1 x-1.5$ respectively. And the theory was greater in field size relative to practical by $0.2 \mathrm{~cm}$ which is within the tolerance limit. Both theoretical and practical field sizes correlation with SSD extension obey the inverse square law as mentioned by Khan [11]. Figure 10: shows the correlation of output in $\mathrm{Gy} / \mathrm{Mu}$ versus field size in $\mathrm{cm}$ at SSD $100 \mathrm{~cm}$. it reveals that: the system output in $\mathrm{Gy} / \mathrm{Mu}$ increases in logarithmic equation form that could be fitted to equation: $y=0.11 \ln (x)+0.732$, where ' $x$ ' refers to field size in $\mathrm{cm}$ and ' $y$ ' refers to system output in $\mathrm{Gy} / \mathrm{Mu}$ as significant correlation $\left(\mathrm{R}^{2}=0.9\right)$. The increasing output as a function of field size increment is ascribed to back scattered radiation and collimator scatters, which added to primary beam [11]. Such exponential trend of output versus field size has been obtained by Masanga [12]. While the correlation between the output in $\mathrm{Gy} / \mathrm{Mu}$ versus $\mathrm{SSD}_{\mathrm{s}}$ at field size of $10 \times 10 \mathrm{~cm}$ revealed that: the system output in $\mathrm{Gy} / \mathrm{Mu}$ has been decreases in logarithmic equation form that could be fitted to $y=$ $1.91 \times \ln (x)+9.846$, where ' $x$ ' refers to SSD in $\mathrm{cm}$ and ' $y$ ' refers to output in $\mathrm{Gy} / \mathrm{Mu}$ which is due to decreased radiation intensity as shown in Figure (11). On the other hand the system output decreases as the SSD increases for both fields $(2 \times 2$ and $10 \times 10)$ which are agreed with the inverse square law (ISL), as well there was significant coincidence between theoretical and practical measurement for both fields $(2 \times 2$ and $10 \times 10)$ as $\left(R^{2}=1\right)$ (Figure 12), these findings are agreed with Khan [13]. The correlation between the output in $\mathrm{Gy} / \mathrm{Mu}$ which represented by ' $y$ ' and the SSD in $\mathrm{cm}$ which represented by ' $x$ ' could be fitted in the equation of the form: $\mathrm{y}=-0.015 x+2.455$ and $y=-0.015 \mathrm{x}+2.499$ for field $10 \times 10$ in practical and theoretical respectively. And $y=-0.009 x+$
1.511 and $y=-0.009 x+1.518$ for field $2 \times 2 \mathrm{~cm}$ in practical and theoretical respectively. One notable finding was that: the measured output on phantom surface was greater $(0.8 \mathrm{~Gy} / \mathrm{MU})$ than that calculated theoretically which was $(0.5$ Gy/MU), which ascribed to effect of back scattered radiation from phantom surface. The influence of temperature and pressure on linear accelerator was studied and revealed that:

The effect of temperature in the reading of dosimeters as fluctuation accuracy and precession is non-avoidable [14], and the general trend in Figure (13) showed that: a significant $\left(\mathrm{R}^{2}=0.8\right)$ reduction in output reading following the increment of temperature and the correlation fitted to equations: $y=-0.0285 x+1.7895$ and $y=-0.032 x+1.5757$ for Linac $10 \mathrm{MV}$ and $6 \mathrm{MV}$ respectively. And as a fact Linac $10 \mathrm{MV}$ showed greater output relative $6 \mathrm{MV}$ throughout the temperature range. The decreasing effects in dosimeter reading following the temperature increment is ascribed to obvious decrement of density [8]. While the effect of pressure is an increasing significant $\left(\mathrm{R}^{2}=0.6\right)$ effect on the output reading (Figure 14) that is ascribed to increment of density by pressure which in turn increases the radiation absorption and hence the dose reading would be higher. However when the correction for the effects of temperature and pressure $\left(\mathrm{K}_{\mathrm{T}, \mathrm{P}}\right)$ applied the dose reading output adjusted to linear horizontal correlation and the significant effects of both $\left(\mathrm{K}_{\mathrm{T}, \mathrm{P}}\right)$ turn to zero i.e. $\left(\mathrm{R}^{2}=0\right)$ as shown in Figure 15 .

For the laser beam alignment, the test revealed that: there is good coincidence in coronal and axial laser; with $1 \mathrm{~mm}$ shift in axial and coronal laser, also $1 \mathrm{~mm}$ in sagittal at the center with tilting in the edge. The penumbra profiles for both Linac 6 and $10 \mathrm{MV}$ (Figures 16) obtained from isodose distribution showed that: an extension of up to 0.5 and $0.3 \mathrm{~cm}$ at maximum depth dose $\left(D_{\max }\right)$ for 1.5 and $2 \mathrm{~cm}$ respectively, however these profiles when assessed by TLD they showed narrow extension as 0.32 and $0.2 \mathrm{~cm}$ for lianc $6 \mathrm{MV}$ and $10 \mathrm{MV}$ respectively. Such variation could be ascribed to TLD sensitivity relative to theoretical measurement using software (ominipro) for treatment planning system [15].

\section{Conclusion}

Linear accelerator has less encountered faults that necessitate the frequent quality control, however for the sake of human live and better care; the QC has to be continued and the most parameters need readjustment are: Gantry angle and the machine isocenter, diaphragm rotation isocenter, the congruence of light and radiation fields and the SSD i.e. all the mechanical parameters.

\section{References}

[1] Indra J. Das, Chee-Wai Cheng, Kashmiri L. Chopra, Raj K. Mitra, Shiv P. Srivastava, Eli Glatstein. (2008). IntensityModulated Radiation Therapy Dose Prescription, Recording, and Delivery: Patterns of Variability Among Institutions and Treatment Planning Systems. J. Natl. Cancer Inst. 100 (5), P: 300 - 307. DOI: 10.1093/jnci/djn020. 
[2] Dische S, Saunders MI, Williams C, Hopkins A, Aird E. (1993). Precision in reporting the dose given in a course of radiotherapy. Radiotherapy Oncology; 29 (3): $287-293$.

[3] ICRU 50. (1993). Prescribing, recording and reporting photon beam therapy, Bethesda, MD: International Commission on Radiation Units and Measurements. ICRU; Report 50.

[4] Hulick PR, Ascoli FA. (2005). Quality assurance in Radiation. J Am Coll. Radiology. 2: 613-616.

[5] Mayles WPM et al. (Eds). (2000). Physics Aspects of Quality Control in Radiotherapy. Report 81, IPEM, York: Institute of Physics and Engineering in Medicine.

[6] Aristoula Papakostidi, Maria Tolia, Nikolaos Tsoukalas. (2014). Quality assurance in Health Services: the paradigm of Radiotherapy. Journal of Balkan Union of Oncology (JBUON). 19 (1): 47-52.

[7] American Association of Physics in Medicine (AAPM). Radiation Therapy Committee Task Group 40. (1994). Comprehensive QA for Radiation Oncology. Report of AAPM Radiation Therapy Committee Task Group 40, Med Phys. 21: 581-618.

[8] Gilgen R., R. Kleinrahm and W. Wagner. (1994). Measurement and correlation of the (pressure, density and temperature) relation of argon II. Saturated-liquid and saturated-vapour densities and vapour pressures along the entire coexistence curve. The Journal of Chemical Thermodynamics, 26 (4): 399-413.
[9] Lauriston S. Taylor and George Singer. (1932). Air density corrections for $\mathrm{x}$-ray ionization chambers. Bureau of Standards Journal of Research, 8 (3): 385-391.

[10] Subramania Jayaraman and Lawrence H. Lanzl. (2004). Clinical Radiotherapy Physics, $2^{\text {nd }}$. Edition, Springer-Verlag Berlin Heidelberg, German.

[11] Khan Faiz. M. (2010). The physics of radiation therapy, $4^{\text {th }}$. edition, Lippincott Williams \& Wilkins- Philadelphia, PA 19106 USA.

[12] Masanga W., P. Tangboonduangjit, C. Khamfongkhruea and C. Tannanonta. (2016). Determination of small field output factors in 6 and $10 \mathrm{MV}$ flattening filter free photon beams using various detectors, $13^{\text {th }}$. South-East Asian Congress of Medical Physics 2015 (SEACOMP) and Journal of Physics: Conference Series 694, 012027. DOI: 10.1088/17426596/694/1/012027.

[13] Khan Faiz. M. (2003). The physics of radiation therapy, $3^{\text {rd }}$. edition, Lippincott Williams \& Wilkins- Philadelphia, PA 19106 USA.

[14] Desrosiers, M. F., Lin, M., Cooper, S. L., Cui, Y., Chen, K. A. (2006). Study of the irradiation temperature coefficient for Lalanine and DL-alanine dosimeters. Radiat. Prot. Dosim. 120, 235-237.

[15] Metcalfe P, Kron T, Elliott A, Wong T, Hoban P. (1993). Dosimetry of 6-MV x-ray beam penumbra. Med Phys. 20 (5): 1439-1445. 